\title{
Genetic stability of pluripotent stem cells during anti-cancer therapies (Review)
}

\author{
WIKTORIA MARIA SUCHORSKA ${ }^{1-3}$, EWELINA AUGUSTYNIAK $^{1,2}$ and MAGDALENA ŁUKJANOW ${ }^{1}$ \\ ${ }^{1}$ Radiobiology Laboratory, Greater Poland Cancer Centre, 61-866 Poznań; ${ }^{2}$ The Postgraduate School of Molecular Medicine, \\ Medical University of Warsaw, 20-091 Warsaw, ${ }^{3}$ Department of Electroradiology, \\ Poznań University of Medical Sciences, 61-866 Poznań, Poland
}

Received July 31, 2015; Accepted December 10, 2015

DOI: $10.3892 /$ etm.2016.2993

\begin{abstract}
Regenerative medicine is a rapidly growing field that holds promise for the treatment of many currently unresponsive diseases. Stem cells (SCs) are undifferentiated cells with long-term self-renewal potential and the capacity to develop into specialized cells. SC-based therapies constitute a novel and promising concept in regenerative medicine. Radiotherapy is the most frequently used method in the adjuvant treatment of tumorous alterations. In the future, the usage of SCs in regenerative medicine will be affected by their regular and inevitable exposure to ionizing radiation (IR). This phenomenon will be observed during treatment as well as diagnosis. The issue of the genetic stability of SCs and cells differentiated from SCs is crucial in the context of the application of these cells in clinical practice. This review examines current knowledge concerning the DNA repair mechanisms (base excision repair, nucleotide excision repair, mismatch repair, homologous recombination and non-homologous end-joining) of SCs in response to the harmful effects of genotoxic agents such as IR and chemotherapeutics.
\end{abstract}

\section{Contents}

1. Stem cells: Genetic integrity

2. Cell cycle of stem cells and DNA damage recognition

3. DNA repair systems in stem cells

4. Failure of DNA repair systems: Apoptosis

5. Conclusions

Correspondence to: Mrs. Ewelina Augustyniak, Radiobiology Laboratory, Greater Poland Cancer Centre, 15 Garbary Street, 61-866 Poznan, Poland

E-mail: ewelina.augustyniak@wco.pl

Key words: pluripotent stem cells, DNA repair, genetic stability, DNA damage response, ionizing radiation, chemotherapeutics

\section{Stem cells: Genetic integrity}

Stem cells (SCs) have high potential and hold great promise in the rapid development of regenerative medicine. Pluripotent SCs are able to self-renew unrestrictedly. They can differentiate in vitro and in vivo in all cell types deriving from the three germ layers $(1,2)$. This ability makes them useful in cell replacement therapy and the treatment of numerous diseases (3), including diabetes (4), neurodegenerative (5), retinal (6) and cardiac (7) diseases, as well as muscular dystrophy (8). SC therapy raises questions concerning the consequences of their influence on an organism. In vivo studies constitute only a small proportion of all research on SCs (9).

Despite the clearly demonstrated effectiveness of SC-derived therapies, this approach has a number of impediments. The response of SCs and stem-derived cells to ionizing radiation (IR) and chemotherapeutics is a questionable issue, particularly with regard to the increase of cancer morbidity in patients $>50$ years old $(10,11)$. Tumor diseases are frequently diagnosed, particularly in elderly patients often burdened with other diseases. How SC therapies affect the organism during cancer treatment (radiotherapy and/or chemotherapy) remains unknown. Exposure to gamma radiation and cisplatin is known to cause DNA damage in cancer cells. These treatments are intended to deprive cancer cells of multiplication potential, and trigger irreparable DNA damage leading to their death (12). However, knowledge concerning the effects of anticancer therapies on healthy cells, including SCs is limited. The exposure of SCs to IR will be unavoidable during treatment and routine diagnosis using computed tomography, positron emission tomography and single-photon emission computed tomography (13).

An additional difficulty in the application of SCs is the evidence that human-induced pluripotent SCs (hiPSCs) and human embryonic SCs (hESCs) are prone to genetic instability during in vitro culture. Frequently chromosomal rearrangements, aneuploidy or defective DNA methylation in both cell types are observed. This results in decreased differentiation capacity and increased proliferation rate (14). Cellular stress, such as freeze-thaw cycles, causes them to be more prone to gene mutation. Manipulation of culture conditions in vitro may contribute to epigenetic instability. Although the majority of cell lines retain a normal karyotype during multiple passages, 
long-term culture increases the risk of anomalies (15). It has also been reported that the process of reprogramming leads to the creation of genetically unstable induced pluripotent SCs (iPSCs). Chromosomal abnormalities in those cells occur at the very early passages (16). The first reports involving abnormal karyotypes of hESCs concerned trisomy of chromosome 12. Chromosomal aberrations may apply to all chromosomes or occur at subchromosomal level. Many of them are also observed in iPSCs (17). Trisomy of chromosome 8 occurs more frequently in hiPSCs than in hESCs. In turn, trisomy 17 was not identified in hiPSCs, but was present in hESCs (18).

Inzunza et al investigated the karyotypes of three hESC lines. The karyotypes of two of the cell lines did not differ, but in the third a monosomy X was demonstrated (19). Genomic and phenotypic changes may be associated with abnormal functioning of SCs, both in the undifferentiated and differentiated stages. Thus, the issue of genetic stability of SCs and cells differentiated from them is crucial in the context of the application of these cells in clinical trials. Further studies are required to demonstrate that iPSCs have no deleterious effect for patients. A high level of DNA damage disrupts the normal functioning of cells. Changes occurring in DNA play an important role during aging, disease conditions and cancer development (20). Specialized repair mechanisms, checkpoints of the cell cycle and tolerance to certain DNA damage protect the integrity of the cell genome, which is required for the normal functioning of cells and their progeny (21). DNA damage is caused by numerous factors, which can arise during replication and transcription, or in response to endogenous and exogenous factors, such as UV radiation, reactive oxygen species, IR and chemical agents (22). However, the nature of the cellular response of SCs to damaging agents and the repair mechanisms remain poorly understood.

The present article provides an overview of the stem and stem-derived cell DNA-damage response to cytotoxic and genotoxic agents during anticancer therapies. Although some research has been carried out on the DNA repair mechanisms of SCs, the complicated mechanisms in undifferentiated, partially differentiated and differentiated cells require elucidation in further studies. The current literature data on DNA repair mechanisms in SCs are explored and discussed in the present review.

\section{Cell cycle of stem cells and DNA damage recognition}

SCs are required to constantly deal with potential damage to their DNA (23). When severe DNA damage occurs, the cell cycle is arrested to prevent aberrant replication, transcription and translation, as well as to preserve energy. However, when DNA damage repair is impossible, cell death mechanisms are activated (24). In response to DNA double-strand breaks (DSBs), ESCs undergo similar events to those of differentiated cells: The kinase ataxia telangiectasia mutated (ATM) becomes phosphorylated at serine 1981 and relocates to DSBs, where it phosphorylates the histone variant protein $\mathrm{H} 2 \mathrm{AX}$ within a few minutes. In mouse embryonic stem cells (mESCs) following exposure to IR, the ATM and ataxia talengiectasia and Rad3-related protein (ATR) kinases phosphorylate $>900$ sites in $\sim 700$ proteins (25). The phosphorylation of histone H2AX is visible as $\gamma \mathrm{H} 2 \mathrm{AX}$ foci. The level of endogenous foci can be also detected in the presence of unrepaired or misrepaired DNA DSBs, the dysfunction of telomeres, genomic instability or senescence. The size of $\gamma \mathrm{H} 2 \mathrm{AX}$ depends on the chromatin structure. During the condensation of chromatin regions, smaller structures can be observed. In turn, when the hyperacetylation of chromatin proceeds or a deficiency of histone $\mathrm{H} 1$ occurs, the $\gamma \mathrm{H} 2 \mathrm{AX}$ foci create bigger structures (26). Hundreds of much smaller $\gamma \mathrm{H} 2 \mathrm{AX}$ foci appear in differentiated cells, although these small foci probably are unrelated to the DNA damage response (27). Moreover, mESCs reveal increased basal levels of $\gamma \mathrm{H} 2 \mathrm{AX}$, even in the absence of DNA DSBs (28). Another important factor is replication protein A, which is single-stranded DNA-binding protein playing a major role in DNA repair pathways (including nucleotide excision, base excision and double-strand break repairs) (29).

The majority of accomplished experiments have been carried out on mESCs. mESCs and hESCs are not equivalent, and differences between them must be taken into consideration. mESCs do not possess the G1 checkpoint and readily undergo tumor protein 53 (p53)-independent apoptosis, as well as demonstrating good repair mechanisms in response to oxidative damage (30). The lack of a functional G1 checkpoint is caused by sequestration of p53 into the cytoplasm. The cells with DNA damage transition from G1 into the S phase, where the lesion can be intensified by a round of replication. mESCs spend $\sim 75 \%$ of their time in S phase, which favors homologous recombination as a main DNA repair mechanism. The p53 protein has an ability to inhibit the activity of homeobox protein Nanog throughout the association with its promoter, which facilitates the differentiation of mESCs. It allows the maintenance of an unchanged population of cells (31). mESCs tolerate only a low level of DNA damage, and for that reason they readily undergo apoptosis or differentiation. As a result of these defensive mechanisms, mESCs generate fewer mutations than somatic cells (32).

The cell cycle in hESCs and mESCs has been found to be shorter in duration than that in somatic cells (Fig. 1A). This is caused by the significantly shorter G1 phase and the enhanced expression of cyclin-dependent kinase 4 (CDK4) and cyclin D2 facilitating transition to the $S$ phase. The defective G1/S checkpoint leads to the accumulation of DNA damage in the $\mathrm{S}$ phase, where DNA damage repair is activated or cell death proceeds (33). hESCs activate cell cycle arrest in G2 and, in contrast to mESCs, p53-dependent apoptosis. Their DNA repair mechanisms are enhanced, which has improved genome protection effects, such as a higher level of DNA-dependent protein kinase catalytic subunit (DNA-PKcs) following irradiation and ultimately more effective repair of DSBs (34). p53 prevents the accumulation of unrepaired DNA lesions throughout cell arrest and DNA repair; in addition, it inhibits the expression of genes responsible for pluripotency, such as Nanog. Studies have indicated that the suppression of p53 may contribute to successful reprogramming. However, in this case the tumorigenicity of iPSCs and their derivatives requires intensive examination (35).

Desmarais et al disclosed that hESCs fail to activate checkpoint kinase (CHK1) and ATM following exposure to cisplatin. Consequently, these cells undergo apoptosis rather than DNA repair. Furthermore, hESCs are able to activate CHK1 or ATM following IR treatment. hESCs and mESCs reveal less oxidative 
A

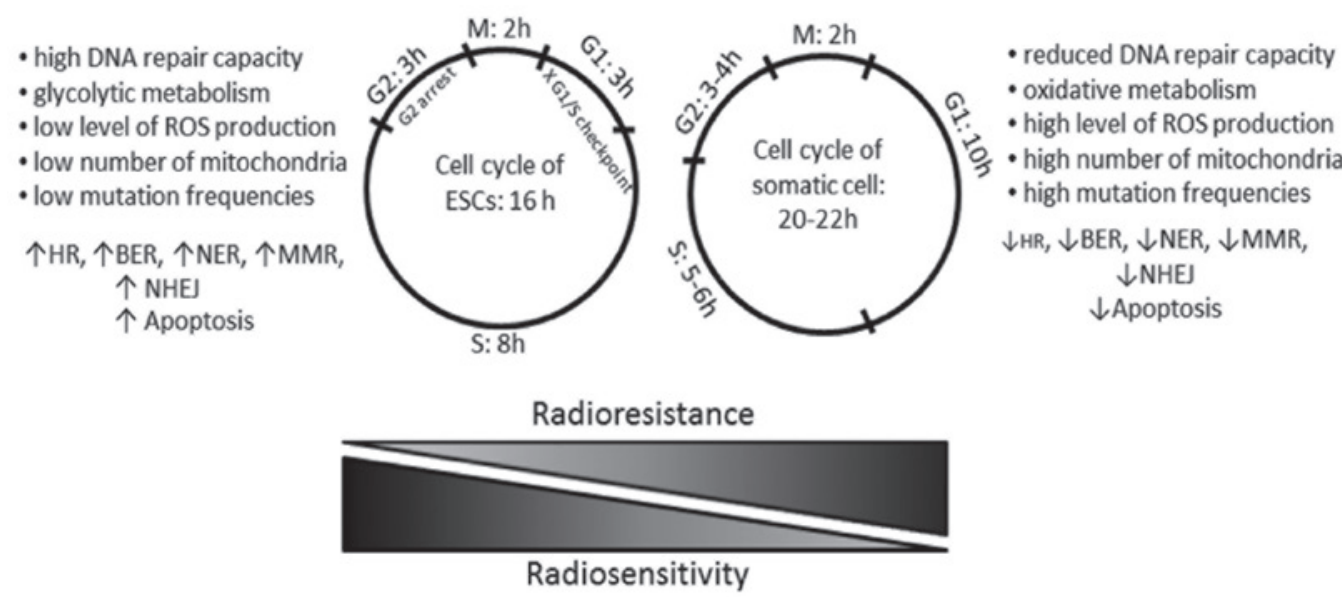

B
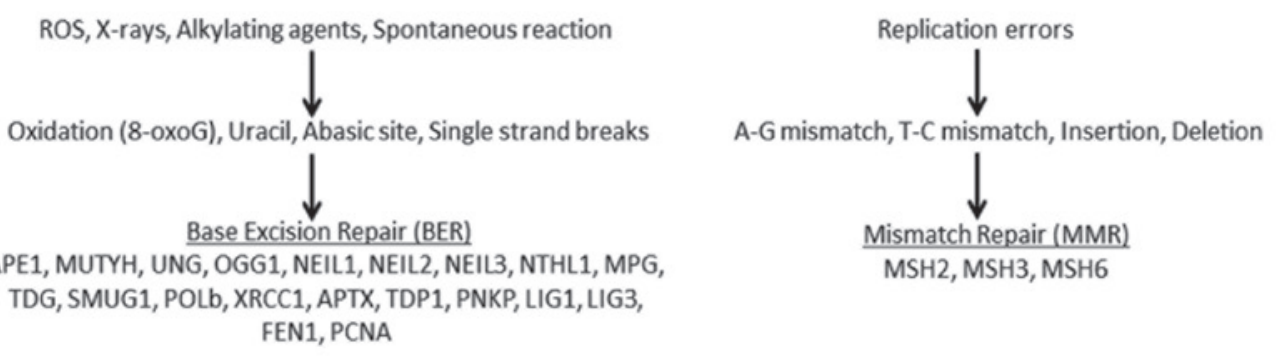

UV light, Polyclic aromatic hydrocarbons
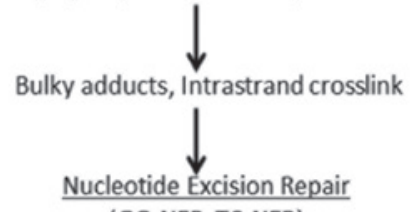

(GG-NER, TC-NER)

XPA, XPB, XPC-RAD23B, XPD, XPE, XPF, XPG, RPA, TFIIX-efectors

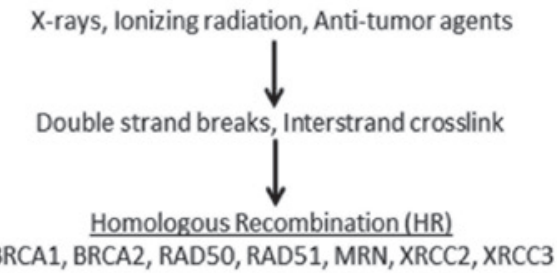

Non- Homologous End Joining (NHEJ)

KU70, KU80, XRCC4, DNA-PKc, DNA ligase IV

C

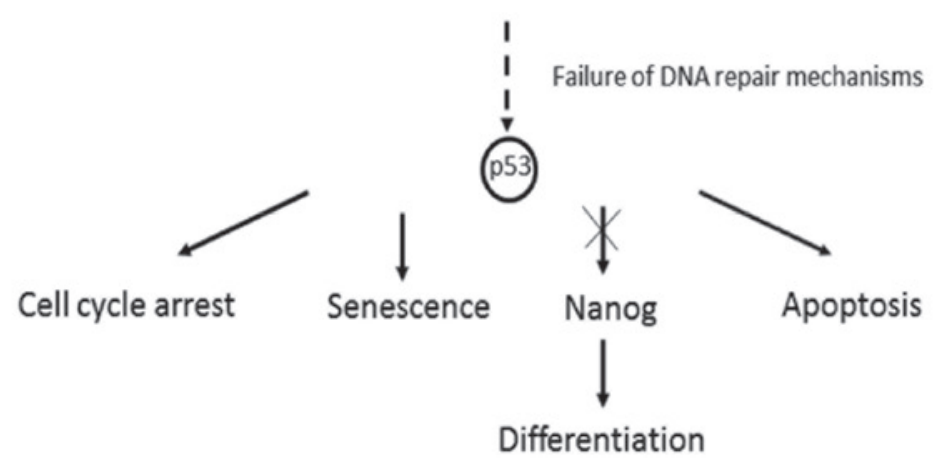

Figure 1. (A) Response of undifferentiated and differentiated cells to genotoxic agents differs, because of distinct cell cycle and cell metabolism. (B) Mammalian cells are exposed to the influence of DNA damage (single- or double-strand breaks) agents. In DNA repair mechanisms, base excision repair, nucleotide excision repair (NER), mismatch repair, homologous recombination and non-homologous end joining are involved many genes. (C) Failure of DNA repair may direct the cells to apoptosis or to differentiation. ESCs, embryonic stem cells; ROS, reactive oxygen species; GG-NER, global genome-NER; TC-NER, transcription-coupled NER.

damage than do differentiated cells (36). This phenomenon may be explained by two concepts. First, ESCs have higher levels of antioxidants that decrease with the progression of differentiation; the downregulation of antioxidant genes results in an increase in the levels of reactive oxygen species. Secondly, mESCs and hESCs demonstrate better DNA repair capacity than differentiated cells such as fibroblasts (37).

\section{DNA repair systems in stem cells}

In accordance with the law of Bergoniè and Tribondeau, cells undergoing intensive division, that are mitotically active or characterized by a high mitotic index, as well as undifferentiated cells, are much more radiosensitive in contrast to other cells. This statement has been confirmed in vitro 
numerous times, with the exception of mature lymphocytes, that although are differentiated, reveal considerably decreased radioresistance (38).

Wilson et al conducted investigations that concerned the irradiation of hESCs cells with low and high doses of gamma radiation. Unsurprisingly, high doses of radiation brought about massive cell death. However, all samples of irradiated hESCs continued to be able to form teratomas, which is a clear test in the determination of pluripotency. It is likely that, although high-dose radiation alters some developmental pathways, it does not influence the expression of pluripotency genes (39). Moreover, hESCs and human mesenchymal SCs (MSCs) are less susceptible to radiation-induced bystander effect signaling than fully differentiated cells in in vitro experiments, which has a direct relevance to cancer therapy (40).

DNA repair pathways. DNA repair is essential for genetic integrity. DNA repair mechanisms can be divided into two main groups: Direct and indirect DNA damage response (DDR). The former includes $\mathrm{O}^{6}$-methylguanine-DNA methyl-transferase (MGMT) activity, which removes methyl from newly created $\mathrm{O}^{6}$ methylguanine adducts; this compound is harmful because it forms complementary base pairs with thymine instead of cytosine, and thus is potentially mutagenic (41). Indirect DNA repair pathways are composed of nucleotide excision repair (NER), base excision repair (BER), mismatch repair (MMR) and DSB recombination repair. DSBs are repaired by error-free homologous recombination (HRR) and error-prone nonhomologous end-joining (NHEJ) (Fig. 1B). The majority of damage triggered by chemo- and radiotherapy is repaired by HRR and NHEJ (42). Numerous proteins involved in the five basic DNA repair mechanisms take part also in apoptosis. In HHR: Breast cancer 1, early onset (BRCA1), ATM, ATR and p53; in NHEJ: DNA-PK; in NER, excision repair cross-complementation group 2 (XPD), p53 and excision repair cross-complementation group 3 (XPB); in BER, poly (ADP-ribose) polymerase 1 (PARP-1), apurinic/apyrimidinic endonuclease/redox effector factor-1 (Ref-1/Ape) and p53; in MMR, mut S homolog 2 (MSH2), Mut S homolog 6 (MSH6) and Mut L homolog 1 (MLH1) (43). This process is discussed later in this review.

DSB repair mechanisms. DSBs cause a massive loss of genetic information, genomic rearrangements and/or cell death. The DSBs are repaired by homologous recombination (HR) and NHEJ. During HR, the undamaged DNA template on the sister chromatid is necessary in order to recover the original sequence. In NHEJ, homology is not required due to modification and ligation of the DNA ends. It often results in deletion or insertions. HR is active in $\mathrm{S} / \mathrm{G} 2 / \mathrm{M}$ phases and NHEJ in G1/S/G2/M phases $(44,45)$. RAD51 and BRCA1 are the most common biomarkers for HR, and DNA-PKcs for NHEJ (46). The RAD51 gene encodes a recombinase protein that induces DNA strand exchange during HR. It plays a pivotal role in DSBs during replication, because RAD51 associates with chromatin during the $\mathrm{S}$ phase and interacts with components of the DNA replication apparatus (47). RAD51 is active in three phases of HR: Presynapsis, synapsis and post-synapsis. During the first stage, the presynaptic filament consisting of six RAD51 molecules and a single-strand DNA (ssDNA) filament is formed. In the synapsis phase, RAD51 creates a heteroduplex DNA (D-loop) created by connection of invading substrate and homologous duplex DNA template. During the last phase, RAD51 exposes the 3-OH group required for DNA synthesis throughout dissociation from double-strand DNA (48). DSB repair using a homologous template can involve HR or single-strand annealing (SSA). SSA is always characterized by mutagenic alterations, as it involves the annealing of sequence repeats located near the DSB and as a result, a deletion between these repeats is observed (49). The participation of very precise HR decreases with the progress of differentiation, whereas the contribution of error-prone NHEJ increases. Oliver et al demonstrated that even during the early stages of differentiation, hMSCs are more prone to cell death. This phenomenon may be explained through the commitment of caspases both in apoptosis and cell differentiation (50).

NHEJ repairs exogenous DSBs triggered by radiation exposure or variable diversity joining (VDJ) recombination. The proteins engaged in this process involve formation of a complex from DNA-PK, Ku70 and Ku80 at the lesion, the alignment of short homologies in overhand regions and recruitment of the ligase IV/X-ray repair cross-complementing protein 4 (XRCC4) complex (20). The Ku complex, due to its toroidal shape, is able to detect DNA damage, maintain the two DNA ends in proximity, inhibit or regulate the activity of exonuclease, and also recruit DNA-PKcs polymerase and ligase (51). DNA-PKcs is the largest known protein with serine/ threonine kinase activity. DNA-PKcs and $\mathrm{Ku}$ bind together to form a DNA duplex. Then, autophosphorylation occurs at $>15$ sites. This activated protein induces the ligase activity of ligase IV/XRCC4. Positive feedback loops are evident: The presence of DNA ligase IV/XRCC4 evokes the autophosphorylation activity of DNA-PKcs. The DNA-PKcs takes part in the regulation of the endonucleolytic activities of artemis, which is another relevant factor for NHEJ (52). Artemis is hyperphosphorylated by the ATM kinase in response to IR. The artemis-DNA-PKcs complex excises damaged DNA overhangs due to its 5' and $3^{\prime}$ endonuclease activity. Moreover, artemis possesses an apparent 5 ' exonuclease activity (53).

DSB repair is more precise in hESCs than it is in somatic human cells. The differentiation of hESCs into astrocytes results in a reduction in the efficiency and fidelity of DSB repair mechanisms. hESCs use homologous recombinational repair rather than NHEJ, which is contrary to the situation in other types of human cells. In addition, a reduction in the accuracy of NHEJ is observed during differentiation. Hence, NHEJ in hESCs is likely to be independent on ATM, DNA-PKcs and PARP as well as dependent on XRCC4 with considerable effectiveness (13). Zou et al examined the response of hESCs and hESC-derived neuronal stem cells (NSCs) to IR (54). They reported that hESCs have DNA repair mechanisms comparable with those of somatic cells, which was not in accordance with other literature data, and revealed that following high-dose treatment, $90 \%$ of irradiated hESCs underwent apoptosis. Following differentiation, the DNA repair capacity of stem-derived cells was found to decline and a higher level of reactive oxygen species was shown. In contrast to hESCs, a sizeable fraction of the NSCs survived high doses of IR (54). In conclusion, although hESCs demonstrate robust DNA repair capacity and tolerate stress, 
they seems to be considerably more sensitive to IR than are the cells differentiated from them.

Sokolov and Neumann investigated the dynamics of transcriptional changes of IR-responsive genes, such as cyclin-dependent kinase inhibitor 1A (CDKN1A/p21/Cip1), growth arrest and DNA-damage-inducible $\alpha$ (GADD45A), proliferating cell nuclear antigen (PCNA), BTG family member 2 (BTG2), BCL2-binding component 3 (BBC3), sestrin 1 (SESN1), DNA damage-binding protein 2 (DDB2), immediate early response 5 (IER5), polo-like kinase 3 (PLK3) and growth differentiation factor 15 (GDF15) during the 'early' and 'late' radioresponse of hESCs. The activation of stress genes exhibited a dose-response correlation, although not in clear linear manner. This may indicate the existence of a threshold for changes in gene expression in certain human ESC genomes for low doses of IR (55). CDKN1A is one of the principal cyclin-dependent kinase inhibitors necessary for effective activation of the G1/S checkpoint following IR treatment in majority of human cells in vitro. However, in response to IR, hESCs and iPSCs fail to induce the G1/S checkpoint. This phenomenon may be caused by reduced $\mathrm{p} 21$ protein levels in hESCs/iPSCs following IR (56). However, the G1/S checkpoint is characteristic for NHEJ not for HR.

Single-strand break repair mechanisms. The mismatch repair (MMR) pathway is responsible for the repair of mismatches created between bases on complementary DNA strands. The mismatches are formed due to polymerase slippage during replication that initiates errors and alterations in nucleotide incorporation, as well as short insertions and deletions (indels). Deficiencies of MMR may induce the accumulation of mutations and microsatellite instability, which is characteristic for hereditary nonpolyposis colon cancer (57). The study carried out by Tichy et al indicates that mESCs are characterized by elevated levels of MMR proteins, which correlates with more efficient MMR. Moreover, in these cells, BER protein expression levels are also elevated and better able to repair an oligonucleotide template (58).

The BER pathway covers two main types of repair: Short patch repair dependent upon DNA polymerase $\beta$ and long patch repair dependent upon PCNA. In short patch repair, single nucleotides are first removed and then replaced. The longer mechanism is based on the removal of nucleotides from the damaged strand, DNA synthesis and consequently, ligation. Those mechanisms eliminate base damage caused by oxidation, alkylation or deamination as well as ssDNA breaks (59).

NER is responsible for eliminating a wide variety of bulky, helix-distorting lesions from DNA, such as cisplatin-DNA intrastrand crosslinks. With regard to the mechanical process, BER and NER are very similar; however, the NER pathway is much more complex mechanism. NER involves DNA damage recognition, local opening of the DNA helix around the lesion, excision of a short single-strand segment of DNA spanning the defect, as well as sequential repair synthesis and strand ligation (60). The NER pathway consists of two subgroups, termed global genome NER (GG-NER) and transcription-coupled NER (TC-NER). The predominant damage recognition factor of GG-NER is the XP complementation group C (XPC)/ $\operatorname{Rad} 23$ homolog B (HR23B)/Centrin (CEN2) system. This system eliminates or reduces DNA lesions throughout the genome.
TC-NER damage recognition includes the arrest of elongating RNA polymerase II (RNAPII) and two proteins: Cockayne syndrome A (CSA) and B (CSB). The XPC complex, CSA and $\mathrm{CSB}$ recruit 10 protein complexes and the multi-functional transcription factor TFIIH to the site of the lesion (61). Undifferentiated cells have higher efficacy of NER and BER than non-pluripotent cells. The GG-NER, but not TC-NER repair mechanism, is superior in human undifferentiated cells in comparison with fibroblasts (62).

\section{Failure of DNA repair systems: Apoptosis}

Failure of DNA repair processes directs cells to undergo apoptosis-programmed cell death (63). Dying cells send signals to neighboring and living cells, inducing the proliferation of stem and progenitor cells. This phenomenon is crucial for the regenerative process (64). The main reason for apoptosis is a loss of genomic integrity caused by the accumulation of DNA damage (65). Although SCs have very efficient DNA repair mechanisms, the lack of a G1 checkpoint causes them to be hypersensitive to IR and other DNA-damaging agents, which facilitates their apoptosis (66). However, the final decision regarding the induction of apoptosis and/or cell cycle arrest is dependent on the magnitude and duration of the damage stimuli (67). In mammalian cells, two main pathways initiating apoptosis exist: Extrinsic and intrinsic (mitochondrial). The key mechanism in both is based on the activation of enzymes known as caspases (68). Caspases can be divided into initiator (caspase-8, -9 and -12) and effector (caspase-3, -6 and -7) types $(69,70)$. An important role in the process of apoptosis is played by $\mathrm{p} 53$. In physiological conditions, the concentration of p53 in the cytosol is low. In response to cellular stress, p53 is accumulated, which leads to the activation of apoptosis (71).

The extrinsic apoptotic pathway is based mainly on binding of death receptor ligand to the receptor membrane. These death receptors include the tumor necrosis factor (TNF) receptor superfamily of TNF-Fas (Apo-1, CD95), TNF receptor 1 (TNF-R1), death receptor 3 (DR-3, Apo-3, TRAMP, WSL-1, LARD), death receptor 4 (TRAIL-R1, DR-4), death receptor 5 (TRAIL-R2, DR-5), death receptor 6 (DR-6), ectodysplasin receptor (EDA-R) and nerve growth receptor (NGF-R). All death receptors are composed of an extracellular domain, transmembrane portion and cytoplasmic death domain (DD), which is necessary for interaction with the adapter protein complex. Receptor-ligand binding leads to a conformational change in the receptor internal domain and formation of the death-inducing signaling complex (DISC), consisting of a death receptor adapter protein known as Fas-associated protein with death domain (FADD) and procaspase- 8 or procaspase- 10 . As a result of DISC dimerization, the activation of caspase- 8 or -10 proceeds. Activated caspases initiate a cascade of reactions leading to cell death (72-74).

The intrinsic apoptotic pathway is associated with the mitochondria and activated by DNA damage or cytotoxic drugs, which triggers changes within the mitochondria, such as increased permeability of the mitochondrial membrane and outflow of cytochrome $c$ to the cytoplasm. Subsequently, cytochrome $c$ activates apoptosis-inducing protein (Apaf-1) and procaspase-9. An apoptosome is formed, which induces other effector caspases (75). Membrane proteins belonging 
to the B-cell lymphoma 2 (Bcl-2) family are involved in the regulation of this process. Some of them: Bcl-2 and B-cell lymphoma-extra large $(\mathrm{Bcl}-\mathrm{xL})$ are anti-apoptotic and increase the likelihood of cell survival. Others, such as Bcl-2-associated $\mathrm{X}$ protein (Bax) and $\mathrm{Bcl}-2$ homologous antagonist killer (Bak), direct the cell to programmed death (76). Although the intrinsic and extrinsic pathways act in an independent manner, the receptor pathway can be associated with the internal pathway via $\mathrm{BH} 3$ interacting domain death agonist (Bid) protein. Caspase- 8 is capable of proteolysis of the pro-apoptotic protein, Bid, which is translocated to the mitochondrial surface. This results in release of cytochrome $c$ and activation of the intrinsic pathway of apoptosis (77).

Mechanism of apoptosis in pluripotent SCs. The mechanism of apoptosis varies, according to cell type and the nature of the stimulus. Filion et al showed that in the majority of hESCs undergoing apoptosis due to DNA damage, apoptosis was directed through the mitochondrial pathway. In stress conditions, the accumulation of $\mathrm{p} 53$ contributes to the inhibition of octamer-binding transcription factor 4 (Oct-4) and Nanog expression, and the induction of spontaneous differentiation (Fig. 1C). This suggests that the utilization of this mechanism ensures the genomic integrity of hESCs (78). In comparison with hESCs, mESCs have lower levels of apoptosis and a reduced capacity for differentiation (79). However, mESCs exposed to DNA damage-inducing UV radiation also reveal a certain degree of ability to induce differentiation by suppressing the expression of Nanog (80). HESCs have a high capacity for DNA repair and a considerable threshold of apoptosis induction compared with differentiated cells. iPSCs exhibit an inferior apoptosis response to reactive oxygen species in comparison with hESCs (32). SCs isolated from the small intestine are sensitive to DNA damage-induced cell death due to their low expression levels of the anti-apoptotic Bcl-2 protein. By contrast, colon SCs are more resistant to radiation; they express high levels of $\mathrm{Bcl}-2$. This demonstrates that the sensitivity to DNA damage and p53-induced apoptosis differs significantly among different types of SCs (81). In conclusion, apoptosis constitutes a defensive mechanism that protects entire populations of cells against the effects of endo-exogenous factors.

\section{Conclusions}

In conclusion, $\mathrm{SCs}$ possess a unique cell cycle, which has a DDR-enhancing effect. The DNA repair mechanisms in pluripotent SCs are more efficient than those in differentiated cells. In particular, HR is favored as a main repair mechanism of DNA DSBs. Unrepaired DNA damage in pluripotent SCs readily directs them to programmed cell death or differentiation. This phenomenon prevents the accumulation of mutations and contributes to the genetic stability of SCs. The genetic integrity of pluripotent SCs and their derivatives is very relevant due to the unavoidable exposure of SCs to genotoxic and cytotoxic agents during diagnostic procedures, as well as during anti-cancer therapies. For that reason, further studies concerning the safety of stem and stem-derived cells treated with IR and/or chemotherapeutics are required.

\section{Acknowledgements}

The present study was supported by the National Science Centre (grant number 2012/07/E/NZ3/01819).

\section{References}

1. Tomizawa M, Shinozaki F, Sugiyama T, Yamamoto S, Sueishi M and Yoshida T: Activin A maintains pluripotency markers and proliferative potential of human induced pluripotent stem cells. Exp Ther Med 2: 405-408, 2011.

2. Lach M, Trzeciak T, Richter M, Pawlicz J and Suchorska WM: Directed differentiation of induced pluripotent stem cells into chondrogenic lineages for articular cartilage treatment. J Tissue Eng 5: 2041731414552701, 2014.

3. Fragma AM, de Araújo ÉSS, Vergani N, Fonseca SAS and Pereira LV: Use of human embryonic stem cells in therapy. In: Stem Cells and Cell Therapy. Al-Rubeai M and Naciri M (eds). Springer, Dordrecht, pp1-19, 2014.

4. Domínguez-Bendala J, Lanzoni G, Inverardi L and Ricordi C: Concise review: Mesenchymal stem cells for diabetes. Stem Cells Transl Med 1: 59-63, 2012.

5. Feng Z and Gao F: Stem cell challenges in the treatment of neurodegenerative disease. CNS Neurosci Ther 18: 142-148, 2012.

6. Jin $\mathrm{ZB}$, Okamoto $\mathrm{S}$, Mandai $\mathrm{M}$ and Takahashi M: Induced pluripotent stem cells for retinal degenerative diseases: A new perspective on the challenges. J Genet 88: 417-424, 2009.

7. Bolli R, Chugh AR, D-Amario D, Loughran JH, Stoddard MF, Ikram S, Beache GM, Wagner SG, Leri A, Hosoda T, et al: Cardiac stem cells in patients with ischaemic cardiomyopathy (SCIPIO): Initial results of a randomised phase 1 trial. Lancet 378: 1847-1857, 2011.

8. Abujarour R, Bennett M, Valamehr B, Lee TT, Robinson M, Robbins D, Le T, Lai K and Flynn P: Myogenic differentiation of muscular dystrophy-specific induced pluripotent stem cells for use in drug discovery. Stem Cells Transl Med 3: 149-160, 2014.

9. Drummond RJ, Kunath T, Mee PJ and Ross JA: Induced pluripotent stem cell technology and stem cell therapy for diabetes. Exp Ther Med 2: 3-7, 2011.

10. de Magalhães JP: How ageing processes influence cancer. Nat Rev Cancer 13: 357-365, 2013.

11. Zaman MH: The role of engineering approaches in analysing cancer invasion and metastasis. Nat Rev Cancer 13: 596-603, 2013.

12. Baskar R, Lee KA, Yeo R and Yeoh KW: Cancer and radiation therapy: Current advances and future directions. Int J Med Sci 9: 193-199, 2012.

13. Sokolov MV and Neumann RD: Human embryonic stem cells responses to ionizing radiation exposures: Current state of knowledge and future challenges. Stem Cells Int 2012: 579104, 2012.

14. Nguyen HT, Geens M and Spits C: Genetic and epigenetic instability in human pluripotent stem cells. Hum Reprod Update 19: 187-205, 2013.

15. Lund RJ, Närvä E and Lahesmaa R: Genetic and epigenetic stability of human pluripotent stem cells. Nat Rev Genet 13: 732-744, 2012.

16. Martins-Taylor K, Nishler SB, Taapken SM, Compton T, Crandall L, Montgomery KD, Lalande M and Xu RH: Recurrent copy number variations in human induced pluripotent stem cells. Nat Biotechnol 29: 488-491, 2011.

17. Peterson SE and Loring JF: Genomic instability in pluripotent stem cells: Implications for clinical applications. J Biol Chem 289: 4578-4584, 2014.

18. Taapken SM, Nisler BS, Newton MA, Sampsell-Barron TL, Leonhard KA, Mclntire EM and Montgomery KD: Karyotypic abnormalities in human induced pluripotent stem cells and embryonic stem cells. Nat Biotechnol 29: 313-314, 2011.

19. Inzunza J, Sahlén S, Holmberg K, Strömberg AM, Teerijoki H, Blennow E, Hovatta $\mathrm{O}$ and Malmgren $\mathrm{H}$ : Comparative genomic hybridization and karyotyping of human embryonic stem cells reveals the occurrence of an isodicentric X chromosome after long-term cultivation. Mol Hum Reprod 10: 461-466, 2004.

20. Kenyon $\mathbf{J}$ and Gerson SL: The role of DNA damage repair in aging of adult stem cells. Nucleic Acids Res 35: 7557-7565, 2007.

21. Giglia-Mari G, Zotter A and Vermeulen W: DNA damage response. Cold Spring Harb Perspect Biol 3: a000745, 2011. 
22. Jackson SP and Bartek J: The DNA-damage response in human biology and disease. Nature 461: 1071-1078, 2009.

23. Blanpain C, Mohrin M, Sotiropoulou PA and Passegué E: DNAdamage response in tissue-specific and cancer stem cells. Cell Stem Cell 8: 16-29, 2011.

24. von Stechow L, Ruiz-Aracama A, van de Water B, Peijnenburg A Danen $\mathrm{E}$ and Lommen A: Identification of cisplatin-regulated metabolic pathways in pluripotent stem cells. PLoS One 8: e76476, 2013

25. Pines A, Kelstrup CD, Vrouwe MG, Puigvert JC, Typas D, Misovic B, de Groot A, von Stechow L, van de Water B, Danen EH, et al: Global phopshoproteome profiling reveals unanticipated networks responsive to cisplatin treatment of embryonic stem cells. Mol Cell Biol 31: 4964-4977, 2011.

26. Banáth JP, Bañuelos CA, Klokov D, MacPhail SM, Lansdorp PM and Olive PL: Explanation for excessive DNA single-strand breaks and endogenous repair foci in pluripotent mouse embryonic stem cells. Exp Cell Res 315: 1505-1520, 2009.

27. Han J, Hendzel MJ and Allalunis-Turner J: Quantitative analysis reveals asynchronous and more than DSB-associated histone $\mathrm{H} 2 \mathrm{AX}$ phosphorylation after exposure to ionizing radiation. Radiat Res 165: 283-292, 2006.

28. Middel V and Blattner C: DNA repair in embryonic stem cells. In: DNA Repair - On the Pathways to Fixing DNA Damage and Errors. Storici F (ed). InTech, Rijeka, Croatia, pp357-380, 2011.

29. Prendergast ÁM, Cruet-Hennequart S, Shaw G, Barry FP and Carty MP: Activation of DNA damage response pathways in human mesenchymal stem cells exposed to cisplatin or $\gamma$-irradiation. Cell Cycle 10: 3768-3777, 2011

30. Luo LZ, Gopalakrishna-Pillai S, Nay SL, Park SW, Bates SE, Zeng X, Iverson LE and O-Connor TR: DNA repair in human pluripotent stem cells is distinct from that in non-pluripotent human cells. PLoS One 7: e30541, 2012.

31. Tichy ED and Stambrook PJ: DNA repair in murine embryonic stem cells and differentiated cells. Exp Cell Res 314: 1929-1936, 2008.

32. Fan J, Robert C, Jang YY, Liu H, Sharkis S, Byalin SB and Rassool FV: Human induced pluripotent cells resemble embryonic stem cells demonstrating enhanced levels of DNA repair and efficacy of nonhomologous end-joining. Mutat Res 713: 8-17, 2011

33. Rocha CRR, Lerner LK, Okamoto OK, Marchetto MC and Menck CFM: The role of DNA repair in the pluripotency and differentiation of human stem cells. Mutat Res 752: 25-35, 2013.

34. Harfouche G and Martin MT: Response of normal stem cells to ionizing radiation: A balance between homeostasis and genomic stability. Muat Res 704: 167-174, 2010.

35. Zhao T and Xu Y: p53 and stem cells: New developments and new concerns. Trends Cell Biol 20: 170-175, 2010.

36. Desmarais JA, Hoffmann MJ, Bingham G, Gagou ME, Meuth M and Andrews PW: Human embryonic stem cells fail to activate CHK1 and commit to apoptosis in response to DNA replication stress. Stem Cells 30: 1385-1393, 2012.

37. Maynard S, Swistowska AM, Lee JW, Liu Y, Liu ST, Da Cruz AB, Rao M, de Souza-Pinto NC, Zeng X and Bohr VA: Human embryonic stem cells have enhanced repair of multiple forms of DNA damage. Stem Cells 26: 2266-2274, 2008.

38. Christensen DM, Iddins CJ and Sugarman SL: Ionizing radiation injuries and illnesses. Emerg Med Clin North Am 32: 245-265, 2014.

39. Wilson KD, Sun H, Huang M, Zhang WY, Lee AS, Li Z, Wang SX and $\mathrm{Wu} \mathrm{JC}$ : Effects of ionizing radiation on self-renewal and pluripotency of human embryonic stem cells. Cancer Res 70 5539-5548, 2010.

40. Sokolov MV and Neumann RD: Radiation-induced bystander effects in cultured human stem cells. PLoS One 5: e14195, 2010.

41. Park Y and Gerson SL: DNA repair defects in stem cell function and aging. Annu Rev Med 56: 495-508, 2005.

42. Maugeri-Saccà $M$, Bartucci $M$ and De Maria R: DNA damage repair pathways in cancer stem cells. Mol Cancer Ther 11 1627-1636, 2012

43. Bernstein C, Bernstein H, Payne CM and Garewal H: DNA repair/pro-apoptotic dual-role proteins in five major DNA repair pathways: Fail-safe protection against carcinogenesis. Mutat Res 511: 145-178, 2002

44. Lundin C, Erixon K, Arnaudeau C, Schultz N, Jenssen D, Meuth $\mathrm{M}$ and Helleday T: Different roles for nonhomologous end joining and homologous recombination following replication arrest in mammalian cells. Mol Cell Biol 22: 5869-5878, 2002.
45. Mao Z, Bozzella M, Seluanov A and Gorbunova V: DNA repair by nonhomologous end joining and homologous recombination during cell cycle in human cells. Cell Cycle 7: 2902-2906, 2008.

46. Lund PK: Fixing the breaks in intestinal stem cells after radiation: A matter of DNA damage and death or DNA repair and regeneration. Gastroenterology 143: 1144-1147, 2012.

47. Serrano L, Liang L, Chang Y, Deng L, Maulion C, Nguyen S and Tischfield JA: Homologous recombination conserves DNA sequence integrity throughout the cell cycle in embryonic stem cells. Stem Cells Dev 20: 363-374, 2011

48. Krejci L, Altmannova V, Spirek M and Zhao X: Homologous recombination and its regulation. Nucleic Acids Res 40: $5795-5818,2012$

49. Fung $H$ and Weinstock DM: Repair at single targeted DNA double-strand breaks in pluripotent and differentiated human cells. PLoS One 6: e20514, 2011.

50. Oliver L, Hue E, Séry Q, Lafargue A, Pecqueur C, Paris F and Vallette FM: Differentiation-related response to DNA breaks in human mesenchymal stem cells. Stem Cells 31: 800-807, 2013.

51. Downs JA and Jackson SP: A means to a DNA end: The many roles of Ku. Nat Rev Mol Cell Biol 5: 367-378, 2004.

52. Lieber MR: The mechanism of double-strand DNA break repair by the nonhomologous DNA end joining pathway. Annu Rev Biochem 79: 181-211, 2010.

53. Wood RD, Mitchell M and Lindahl T: Human DNA repair genes 2005. Mutat Res 577: 275-283, 2005.

54. Zou Y, Zhang N, Ellerby LM, Davalos AR, Zeng X, Campisi J and Desprez DY: Responses of human embryonic stem cells and their differentiated progeny to ionizing radiation. Biochem Biophys Res Commun 426: 100-105, 2012.

55. Sokolov M and Neumann R: Effects of low doses of ionizing radiation exposures on stress-responsive gene expression in human embryonic stem cells. Int J Mol Sci 15: 588-604, 2014.

56. Momcilović O, Choi S, Varum S, Bakkenist C, Schatten G and Navara C: Ionizing radiation induces ataxia telangiectasia mutated-dependent checkpoint signaling and G (2) but not G (1) cell cycle arrest in pluripotent human embryonic stem cells. Stem Cells 27: 1822-1835, 2009.

57. Kunkel TA and Erie DA: DNA mismatch repair. Annu Rev Biochem 74: 681-710, 2005

58. Tichy ED, Liang L, Deng L, Tischfield J, Schwemberger S, Babcock G and Stambrook PJ: Mismatch and base excision repair proficiency in murine embryonic stem cells. DNA Repair (Amst) 10: 445-451, 2011

59. Robertson AB, Klungland A, Rognes $\mathrm{T}$ and Leiros I: DNA repair in mammalian cells: Base excision repair: The long and short of it. Cell Mol Life Sci 66: 981-993, 2009.

60. Shuck SC, Short EA and Turchi JJ: Eukaryotic nucleotide excision repair: From understanding mechanisms to influencing biology. Cell Res 18: 64-72, 2008.

61. Dexheimer TS: DNA repair pathways and mechanisms. In: DNA Repair of Cancer Stem Cells. Mathews LA, Carbarcas SM and Hurt E (eds). Springer International Publishing, Dordrecht, pp19-32, 2013.

62. de Waard H, Sonneveld E, de Wit J, Esvaldt-van Lange R, Hoeijmakers JH, Vrieling $\mathrm{H}$ and van der Horst GT: Cell-type-specific consequences of nucleotide excision repair deficiencies: Embryonic stem cells versus fibroblasts. DNA Repair (Amst) 7: 1659-1669, 2008.

63. Ryoo HD and Bergmann A: The role of apoptosis-induced proliferation for regeneration and cancer. Cold Spring Harb Perspect Biol 4: a008797, 2012

64. Li F, Huang Q, Chen J, Peng Y, Roop DR, Bedford JS and Li CY: Apoptotic cells activate the 'phoenix rising' pathway to promote wound healing and tissue regeneration. Sci Signal 3: ra13, 2010.

65. Vahidi Ferdousi L, Rocheteau P, Chayot R, Montagne B, Chaker Z, Flament P, Tajbakhsh S and Ricchetti M: More efficient repair of DNA double-strand breaks in skeletal muscle stem cells compared to their committed progeny. Stem Cell Res 13: 492-507, 2014.

66. Hong Y and Stambrook PJ: Restoration of an absent G1 arrest and protection from apoptosis in embryonic stem cells after ionizing radiation. Proc Natl Acad Sci USA 101: 14443-14448, 2004

67. Rich T, Allen RL and Wyllie AH: Defying death after DNA damage. Nature 407: 777-783, 2000.

68. He YC, Zhou FL, Shen Y, Liao DF and Cao D: Apoptotic death of cancer stem cells for cancer therapy. Int J Mol Sci 15: 8335-8351, 2014.

69. Fuchs Y and Steller H: Programmed cell death in animal development and disease. Cell 147: 742-758, 2011. 
70. Creagh EM and Martin SJ: Caspases: Cellular demolition experts. Biochem Soc Trans 29: 696-702, 2001.

71. Xu X, Cowley S, Flaim CJ, James W, Seymour L and Cui Z: The roles of apoptotic pathways in the low recovery rate after cryopreservation of dissociated human embryonic stem cells. Biotechnol Prog 26: 827-837, 2010.

72. Yang JK: FLIP as an anti-cancer therapeutic target. Yonsei Med J 49: 19-27, 2008.

73. Würstle ML, Laussmann MA and Rehm M: The central role of initiator caspase-9 in apoptosis signal transduction and the regulation of its activation and activity on the apoptosome. Exp Cell Res 318: 1213-1220, 2012.

74. Mcllwain DR, Berger T and Mak TW: Caspase functions in cell death and disease. Cold Spring Harb Perspect Biol 5: a008656, 2013.

75. Elmore S: Apoptosis: A review of programmed cell death Toxicol Pathol 35: 495-516, 2007.

76. Roos WP and Kaina B: DNA damage-induced cell death: From specific DNA lesions to the DNA damage response and apoptosis. Cancer Lett 332: 237-248, 2013.

77. Igney FH and Krammer PH: Death and anti-death: Tumour resistance to apoptosis. Nat Rev Cancer 2: 277-288, 2002.
78. Filion TM, Qiao M, Ghule PN, Mandeville M, van Wijnen AJ, Stein JL, Lian JB, Altieri DC and Stein GS: Survival responses of human embryonic stem cells to DNA damage. J Cell Physiol 220: 586-592, 2009.

79. Qin H, Yu T, Qing T, Liu Y, Zhao Y, Cai J, Li J, Song Z, Qu X, Zhou P, et al: Regulation of apoptosis and differentiation by p53 in human embryonic stem cells. J Biol Chem 282: 5842-5852, 2007.

80. Neganova I, Vilella F, Atkinson SP, Lloret M, Passos JF, von Zglinicki T, O-Connor JE, Burks D, Jones R, Armstrong L and Lako M: An important role for CDK2 in G1 to S checkpoint activation and DNA damage response in human embryonic stem cells. Stem Cells 29: 651-659, 2011.

81. Mandal PK, Blanpain C and Rossi DJ: DNA damage response in adult stem cells: Pathways and consequences. Nat Rev Mol Cell Biol 12: 198-202, 2011. 